\title{
Effects of Prong-Wire Interferences in Dual Hot-Wire Probes on the Measurements of Unsteady Flows and Turbulence in Low- Speed Axial Fans
}

\author{
Argüelles Díaz, K.M. ${ }^{(1)}$; Fernández Oro, J.M.; \\ Galdo Vega, M.; Blanco Marigorta, E. \\ Universidad de Oviedo, Área de Mecánica de Fluidos. \\ Campus de Viesques, 33271, Gijón (Asturias), Spain. \\ (1) arguelleskatia@uniovi.es
}

\begin{abstract}
Support needles of Dual Hot Wire (DHW) anemometers induce significant inaccuracies for flow angle and turbulence measurements in the case of X-array probes with prongs perpendicular to the flow plane. At certain angular ranges of the incident flow, a wake interference is established between the sensors which leads to a practical limitation of the device. In the case of turbomachinery environments, this is even more critical due to the inherent unsteadiness of the flow direction rotor downstream.

In the present work, the measurement deviation caused by hot-wire probes operated under interference effects has been studied and evaluated, in both steady and unsteady conditions, especially for turbomachinery flows. New designs of DHW probes without prong-wire interference effects in their operative angular ranges were developed for validation. In particular, both V-type and Z-type interference-free probes are compared to a classic X-type probe susceptible for prongwire interferences. Firstly, a steady calibration is performed to show the baseline deviation of the $\mathrm{X}$-array probe in the measurement of the velocity magnitude, the flow angle and the turbulence intensity. Typical errors up to $10-13 \%$ in velocity, 5.5-7 deg in angle and $1.5-2.5$ points overestimation in turbulence levels are observed. Also, unacceptable inaccuracies are found in the turbulence spectra of the measurements.

Following, the impact of the interference for unsteady flow measurements is highlighted comparing the performance of the three probes within the single stage of a low-speed axial fan. The unsteady measurements of the X-array probe have revealed similar averaged discrepancies to those observed in the steady performance, but the instantaneous deviations can be as high as a $20 \%$ in velocity and $16-18$ deg in flow angle in those regions (rotor wakes) with large unsteady velocity gradients and turbulence generation. Turbulence intensity measured in the rotor wakes is also excessively higher.
\end{abstract}

Keywords: hot-wire anemometry, $\mathrm{X}$-array probes, prong-wire interference, time-resolved measurements, turbulence intensity, axial turbomachinery. 


\section{1.- INTRODUCTION}

Thermal anemometry is a measuring technique that retrieves the flow velocity through heat transfer variations in a small sensor that it is electrically heated while exposed to a moving fluid. The most common thermal anemometer is the hot wire thermal device, which it is composed by a single or multiple tiny wires mounted on the probe tip. Every wire is weld to a couple of prongs, usually of stainless steel, incrusted to the probe support, which are also employed as electrical contacts to heat the wire to a temperature in the range of 200-300 $\mathrm{C}$.

Wire's diameter must be extremely reduced for a high frequency response. Typical wire diameters are ranged between 2 to $5 \mu \mathrm{m}$, resulting in extremely low Reynolds numbers which lead to consider the incident flow over the wires as symmetric and quasi-steady. Additionally, the wire's length must be also quite reduced to enlarge the probe's spatial resolution. Optimal values are found when the length-to-diameter ratio is about 200 ([1]), although it must be kept in mind that the spatial resolution is also conditioned by the existing distance between the wires ([2]).

When the flow impinges on a hot wire, part of its heat energy is advected by the flow (forced convection [3]). Because of the wire thermal inertia, its frequency response to flow velocity variations is dumped, so it needs to be electronically compensated using, for instance, a constant temperature anemometer (CTA). This kind of equipment is an amplifier device that feeds back the wires to maintain its temperature independent of the variations in the flow velocity ([4]). Using this technique, the frequency limit can be significantly raised, up to 1000 times higher ([5], [6]).

Every probe must be calibrated before it can be used to measure a particular flow. The calibration determines a relationship between the output voltage of every single wire and the magnitude and direction of the flow velocity vector. A sensitivity function is then introduced to retrieve the velocity magnitude, while an angular calibration provides the directional response of the probe. Depending on the number of wires, pitch and/or yaw variations should be performed to obtain the angular range ([7]).

Using dual hot wire (DHW) probes, we can obtain in-plane velocity components of the flow. The most common configuration is the so-called X-probe (a.k.a. X-array), consisting in a hot wire anemometer with two crossed wires, either in an orthogonal or a non-orthogonal disposition. If the wires are orthogonal to each other, the maximum angular range cannot exceed $90 \mathrm{deg}$, while higher angle between the wires (i.e. $120 \mathrm{deg}$ ) may provide an extended angular range ([8], [9]). Anyway, whatever the angle selected, if the X-probes are built with the supporting prongs in a perpendicular orientation respect to the measuring plane (figure 1), there are certain angular positions where interference effects between the first wire and the prongs of the second wire arise. These effects are superimposed to the self interference of one wire with its own prongs, which is clearly less important than crossed interferences ([10]). 


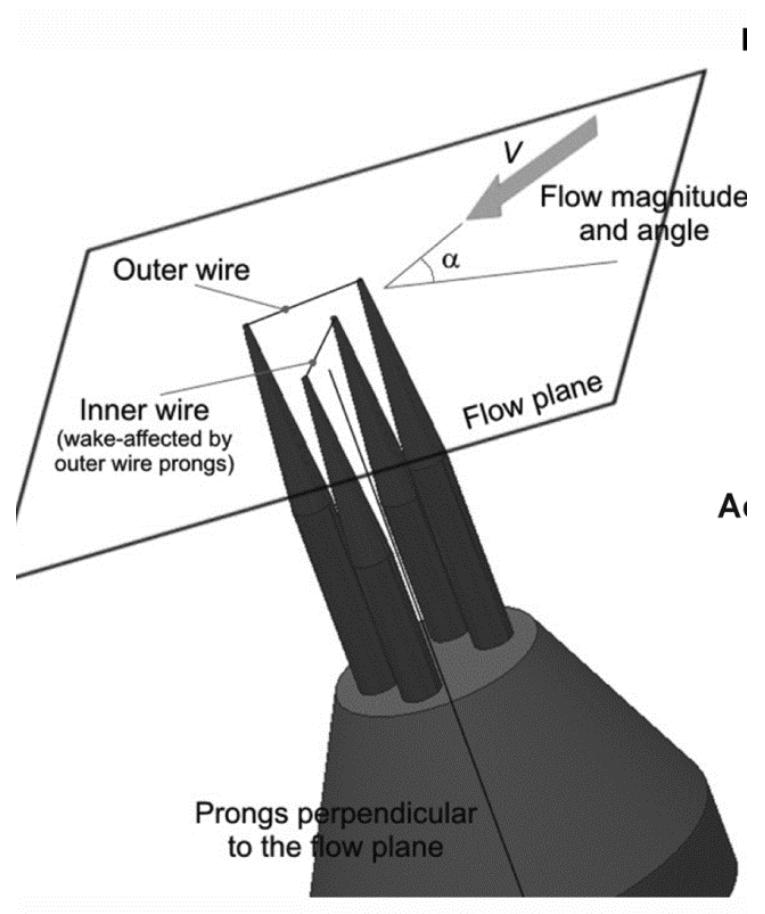

Figure 1. Typical X-type DHW probe with prong-wire interference.

The crossed interference effect appears for certain yaw angles when one of the probe's wires is found in the wake of a prong of the othere. This effect, which has been already identified and studied by several authors ([11], [12]), is known as "wake interference effect", and implies that the wire affected by the prong wake (i.e. in a region with a notable velocity deficit) is measuring flow velocities significantly lower than those of the real flow. Additionally, the measured turbulence level is also higher than the true value because the shedding vortices from the upstream prong are sensed by the downstream wire ([13]). The direct consequence is a reduction in the measuring angular range of the probe and a severe limitation for turbulence measurements. To overcome these problems, the probes can be designed with the supporting prongs placed in a plane parallel to the one of the incident flow. Hence, the wake interference effect vanishes, remaining exclusively the interference effect of the wires with their own prongs, and increasing the angular range accordingly.

For the present investigation, two DHW probes have been built, denoted as Z-type and V-type, with the wire prongs placed in a parallel plane to the flow to avoid interferences. These probes have been compared with a classic 120 deg X-type probe in both steady and unsteady conditions. It is found that both Z-type and V-type calibrations are similar (for probes with identical geometrical characteristics in terms of wires diameter, wires lengths and overall probe size), presenting accurate calibration coefficients. On the other hand, the $\mathrm{X}$-array probe retrieves significant errors up to 10-13\% in velocity, 5.5-7 deg in angle and 1.5-2.5 points overestimation in turbulence levels under steady flow. Additionally, the impact of the interference is analyzed also for unsteady conditions in the case of a low-speed axial fan. In this case, though averaged errors are quite similar with respect to steady deviations (in accordance to bibliography [14]), instantaneous deviations can be as high as a $20 \%$ in velocity and 16-18 deg in flow angle, especially in those regions with large unsteady gradients (rotor wakes). 


\section{2.- PROBES GEOMETRY AND CALIBRATION SETUP}

Three different probe geometries have been considered for the present investigation. The original design is a classical X-type probe with the supporting prongs perpendicular to the flow plane. This means that the inner wire suffers wake interferences coming from the prongs of the outer wire. The remaining probes, designated as Z-type and V-type probes, are improved versions developed to eliminate interference effects, with the prongs placed in a plane parallel to the flow. All the probes were in-home manufactured, with tungsten wires of $5 \mu \mathrm{m}$ and $1 \mathrm{~mm}$ long, in a 120 deg crossed arrangement. The wire prongs are stainless steel needles with $0.4 \mathrm{~mm}$ diameter. Figure 2 presents a picture and a schematic view of the geometrical characteristics of the probes.

X-probe $120^{\circ}$
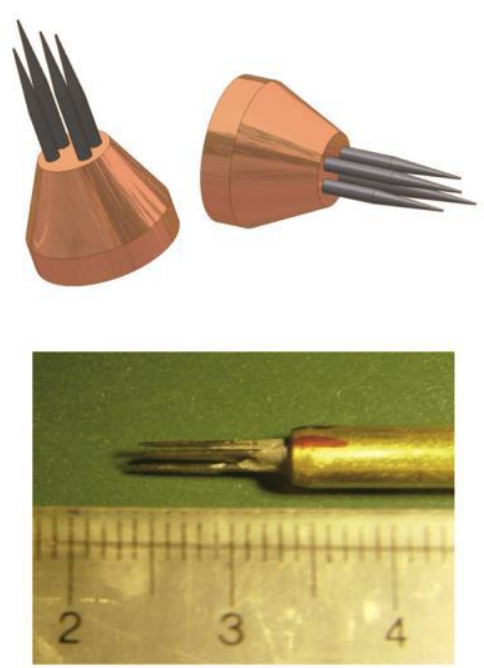

Z-probe $120^{\circ}$


V-probe $120^{\circ}$
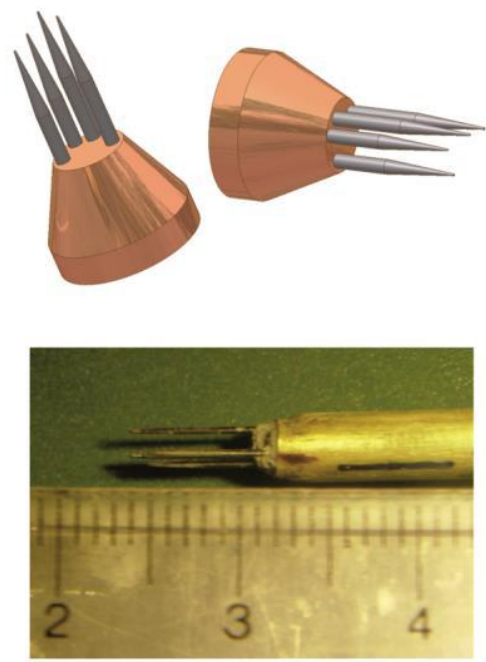

Figure 2. Schematic view of the probes: X-type, Z-type and V-type.

Both Z-type and V-type probes present analogous calibration coefficients and similar measurements results (it is shown later). Hence, for practical reasons, we have employed intensively the $V$-type probe for the experimental characterization of flow fields within the lowspeed fan due to its higher compactness and simplicity to be manufactured and repaired.

The calibration facility is an opened nozzle with a circular discharge section of $1.5 \mathrm{~cm}$ dia. This nozzle supplies a uniform incident flow over the hot-wire probes when mounted in the test section. The flow rate can be regulated with a throttling valve placed on the compressed air supply, so the velocity can be ranged from 0 to $70 \mathrm{~m} / \mathrm{s}$ approximately.

The probes are typically placed $20 \mathrm{~mm}$ downstream of the nozzle discharge, aligned with the jet axis. For a fixed angular position (probe aligned with the incoming flow), the velocity is progressively increased from zero to the maximum velocity, obtaining the output voltage of the wires. Therefore, the response of both wires is directly related to the effective velocity (the velocity component normal to each wire and responsible for the heat transfer). On the other hand, an additional angular calibration is needed to obtain the directional response of the probe. This is done changing the incidence angle of the calibration flow for a fixed velocity magnitude. To that end, a two-axis mechanical gear, driven by a couple of step-by-step motors controlled via PC, 
is employed to carry out this angular repositioning. In this application for a two-wire anemometer, only one axis rotation is needed (yaw angle), while three-wire probes require both yaw and pitch angles relocations. Finally, the effective velocity of every wire is monitored as a function of the flow angle and the angular calibration coefficients of the probe are determined to complete the procedure.
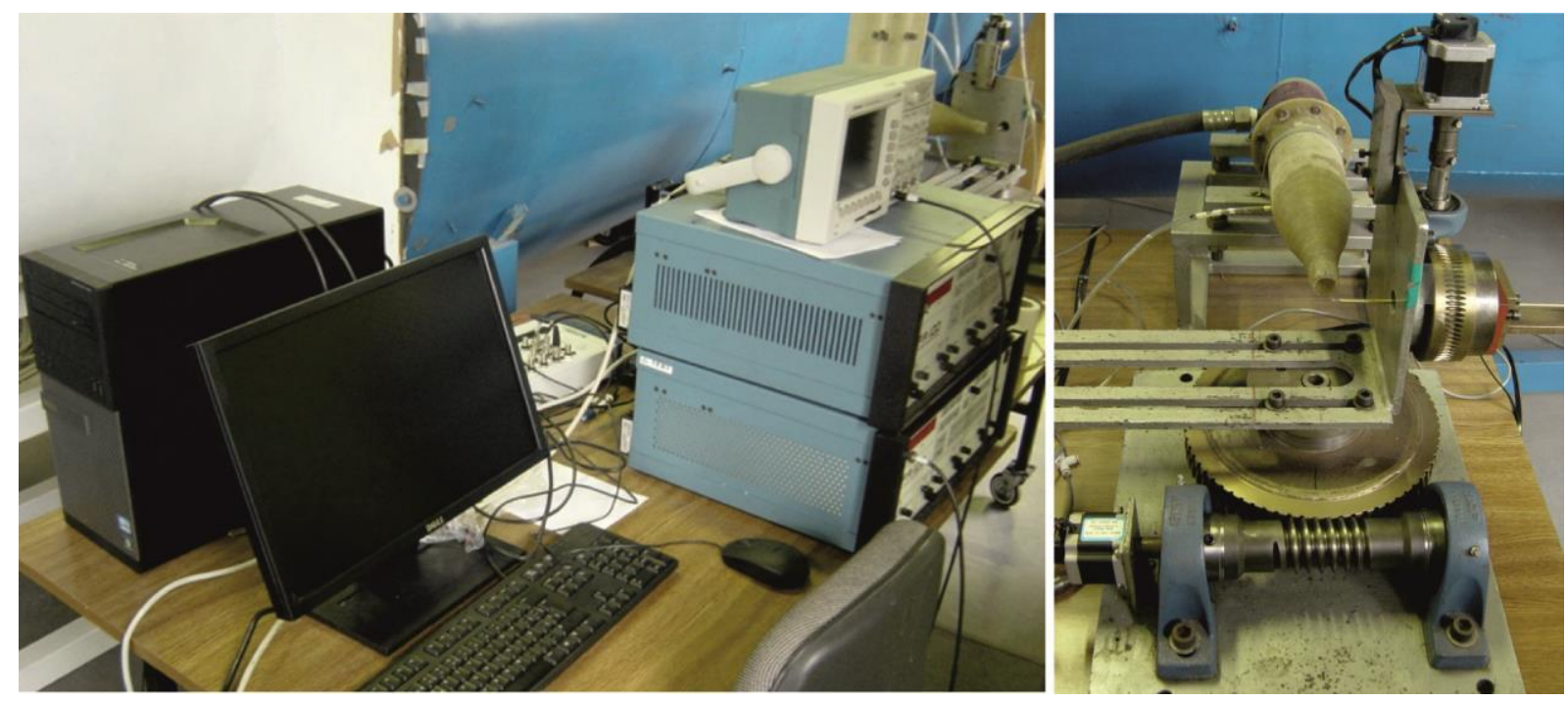

Figure 3. a) Acquisition system: IFA 100 and NIDAQ devices. b) Nozzle and calibration facility.

During the calibration, the probe wires are connected to a four channel, TSI IFA-100, CTA anemometer. The measured data is acquired with a NIDAQ PCI device, 16-bit A/D converter, which are subsequently stored in a PC using MATLAB routines and specific libraries. For every measuring point, up to 104 samples, at a low-frequency response of $100 \mathrm{~Hz}$, are recorded. The surrounding environmental conditions of the calibration facility are controlled and the temperature variations within the calibration room are also registered. Additionally, calibration measurements have been conducted modifying the inlet turbulence of the incident flow (with a perturbing grid mounted on the nozzle) to analyze the impact of this additional parameter on the wake interference effect.

\section{3.- STATIC CALIBRATION AND STEADY PERFORMANCE}

The most popular method to calibrate hot wire probes consists in keeping them steadily in a moving fluid. This calibration, known as "static calibration", is completed in a two-step sequence. In the first one, the CTA output voltage is obtained as a function of the effective velocity in every wire. Following, a mathematical fitting is introduced to link those variables using a transfer curve, like the King's law if the effective velocity is employed ([15]), or a similar sensitivity function. In this case, we have employed a $5^{\text {th }}$ order polynomial fitting with a correction factor for the temperature difference, $U_{\text {eff }}=f\left(E / \sqrt{T_{w}-T_{a}}\right)$, where $U_{\text {eff }}$ is the effective velocity for the wire, $E$ is the output voltage and $T_{w}$ and $T_{a}$ are the wire and air temperatures respectively. 
The second step is to complete the angular calibration, required for two and three-wire probes to determine its directional sensitivity. Typically, three coefficients are employed to represent the flow angle and the change in velocity magnitude ([16]-[18]) for every angular position. Ref. [19] describes in detail a direct calibration procedure for a three-wire probe, including the uncertainty transmitted to the measurements; while references [20] and [21] provide an in-depth review of the different calibration methods available for hot wire probes.

A direct calibration procedure ([19]) has been applied with these three DHW probes, ad hoc manufactured in the Fluid Mechanics Lab of the School of Engineering at the University of Oviedo. For every probe, the sensitivity function has been experimentally determined, and the angular calibration for a reference velocity has been completed. Finally, an angular calibration coefficient, Acf, has been defined from the effective velocities of the wires as follows:

$$
\operatorname{Acf}(\alpha)=\frac{U_{e f f, 1}-U_{e f f, 2}}{\sqrt{U_{e f f, 1}^{2}+U_{e f f, 2}^{2}}}
$$

where $U_{\text {eff, }, 1}$ and $U_{\text {eff, } 2}$ correspond to the effective velocities of both external (wire 1 ) and internal (wire 2) wires, given by the output voltages.

\section{1.- Wake interference effect on the calibration coefficient}

Interference effects between the wires and their own prongs or between the wires and the wake fluid coming from adjacent prongs are manifested as oscillations in the output signals of the probe. Such perturbations may lead to the appearance of two different detrimental effects. On the one hand, they can induce the arising of double points in the distribution of the angular calibration, which implies the impossibility for a unique determination of the angle and a practical reduction in the operative angular range of the probe. On the other hand, if the flow angle to be measured is placed within the angular interval of the calibration affected by interference effects, the measurement accuracy drops severely and consequently the error in the determination of the velocity and flow angle increases.

Precisely, figure 4 shows the angular distributions of the output signals (in voltage) in the wires of the 120 deg X-type probe, obtained during calibration for an angular discretization of 2.5 deg. Note that self-interference of a wire with its own prong is more pronounced for the second wire than for the first one, because of its shorter length. These interference effects are manifested at -45 deg approximately for wire 2 , and at -45 deg for wire 1 . As expected, the wake interference effect is only experienced in the inner wire, because of the velocity deficit induced by the outer wire prongs for yaw angles ranged between 15 and $35 \mathrm{deg}$. Consequently, the operative zone of the angular calibration (60 deg) is established between -45 and $15 \mathrm{deg}$. 


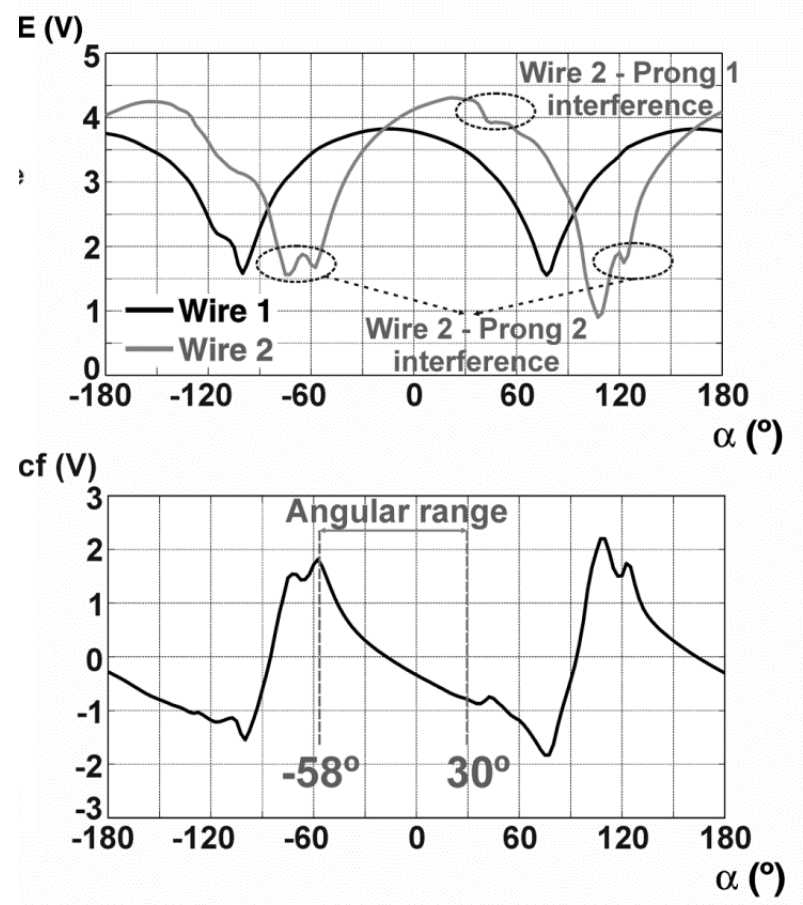

Figure 4. Angular distributions of the wires output voltage (top) and the calibration coefficient (bottom) for the X-array probe.

V-type and Z-type probes are designed to prevent the appearance of wake interferences. Figure 5 (left) shows the angular distributions of the wire's output signals corresponding to these probes. Only self-interactions with their own prongs arise, being more evident for both wires than in the case of the X-type probe. V-probe presents less pronounced effects of self-interference which are now set off at 90 deg for wire 2 and -130 and 60 deg for wire 1 (top plot). Complementarily, Zprobe experiences self-interference at -75 and 80 deg for the first wire and around -22 and 135 deg for the second wire (bottom plot).
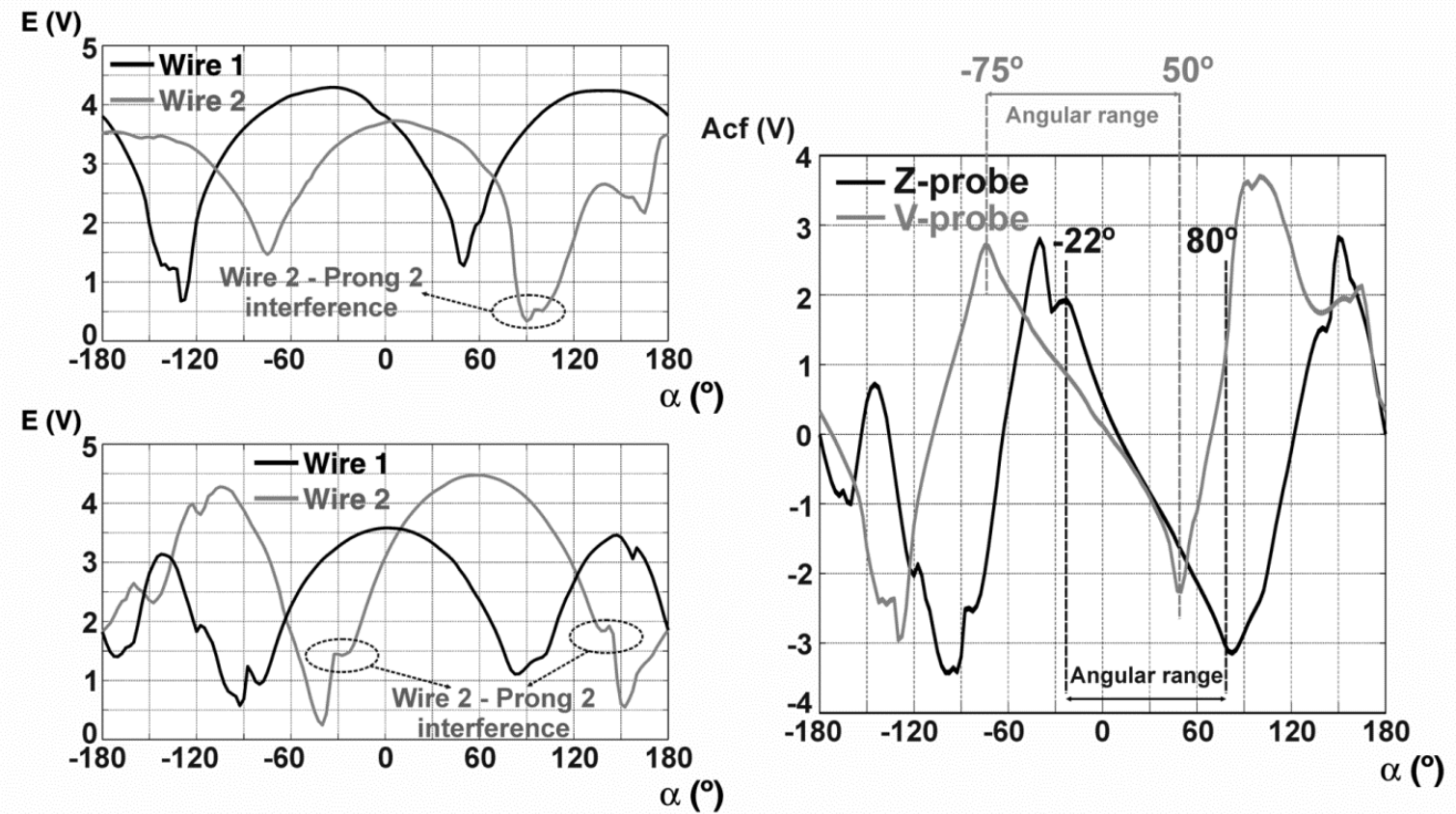

Figure 5. Angular distributions of wires output voltage (left) and calibration coefficients for Z-type and V-type probes (without wake interference, right). 
Additionally, figure 5 (right) represents the angular coefficients of both $\mathrm{V}$ and Z-type probes obtained after using equation 1. The angular range for the Z-type probe turns out to be $102 \mathrm{deg}$, while the V-type is extended to $125 \mathrm{deg}$. In both cases, the significant increment in the operative angular range (with respect to the $88 \mathrm{deg}$ of the X-probe) is a direct consequence of the absence of wake interference effects over the inner wire of the probes. For these free-wake interference probes, the limitation in the angular range comes from the self-interaction of the wires with their own prongs (Z-type probe presents a lower angular range because their self-interactions are more evident).

In order to study how wake interferences have a detrimental effect on the measurements accuracy, several tests with the classic 120 deg X-type probe have been conducted. For that purpose, the starting point has been the determination of the angular calibration for an averaged incident flow velocity of $40 \mathrm{~m} / \mathrm{s}$, already reported in figure 4 . After calibration, the test facility is also employed for measurements acquisition. In particular, measurements have been performed for several flow velocities (differing from the calibration conditions) ranging from 30 to $50 \mathrm{~m} / \mathrm{s}$ at intervals of $10 \mathrm{~m} / \mathrm{s}$. Additionally, we have gone through a wide number of angular positions including free-wake positions and also locations susceptible of wake interference. The incidence flow angle has been ranged from -40 to $40 \mathrm{deg}$ in a $10 \mathrm{deg}$ interval between consecutive positions.

\section{2.- Error in the velocity determination with the X-type probe}

Figure 6 represents the error associated to flow velocity retrievement when the $40 \mathrm{~m} / \mathrm{s}$ calibration is employed to reduce data coming from measurements taken at other flow velocities. The error is expressed in a percentage basis according to the following formula:

$$
e_{v}=100 \frac{\left|v_{\text {real }}-v_{\text {meas }}\right|}{v_{\text {real }}}
$$

where $v_{\text {meas }}$ is the velocity registered with the hot-wire probe and $v_{\text {real }}$ is the real velocity magnitude measured by means of a pressure transducer connected to the NIDAQ acquiring card in the nozzle discharge.

A wake-free region is clearly identified between -40 and 10 deg in figure 6 . There, it is observed a slight trend to obtain higher error for lower velocities than for higher velocities with respect to the calibration reference. Also, minimum errors appear for zero incidence angles, being the lowest around $1 \%$ for $40 \mathrm{~m} / \mathrm{s}$ incident flow. On the contrary, the wake-affected region is evident between 10 and $40 \mathrm{deg}$, where the error in the velocity determination can be as high as a $10-13 \%$. In particular, the error is generally the largest at 20 deg where the interference is maxima; and a similar trend than before, with higher errors for lower velocities, can be concluded. Note that $10 \%$ error corresponds to the interference performance when measuring a flow velocity similar to the calibration magnitude, while $13 \%$ error corresponds to out-of-calibration measurements.

Consequently, when an incident flow angle is measured with the probe working under wakeinterference conditions, the error involved in the determination of the velocity magnitude is excessive to validate that angular region, so the operative range has to be limited to the wake-free regions. 
$120^{\circ}$ DHW X-probe

Angular range with and without interference effects

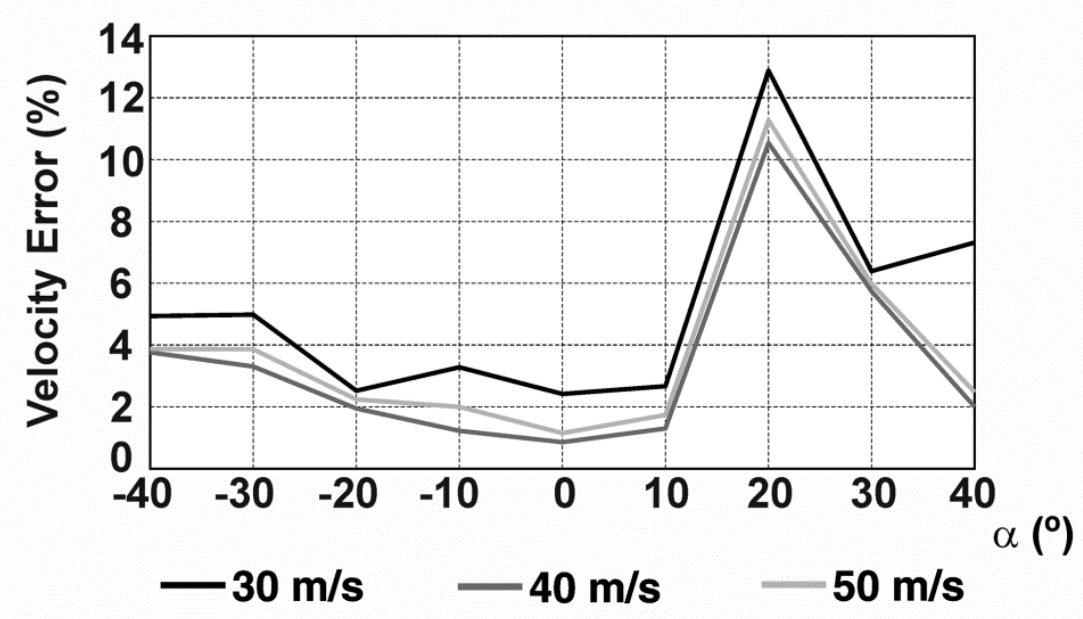

Figure 6. Error associated to the determination of the velocity magnitude at different operating conditions.

\section{3.- Error in the angle determination with the X-type probe}

Following, similar considerations for the flow angle determination are presented below. Now, the error in the angle retrievement when measuring for velocities out of the calibration conditions is calculated in absolute terms according to:

$$
e_{\alpha}=\left|\alpha_{\text {real }}-\alpha_{\text {meas }}\right|
$$

where $\alpha_{\text {meas }}$ represents the flow angle retrieved with the X-type probe and $\alpha_{\text {real }}$ corresponds to real misalignment of the probe, respect to the incident jet, determined from the repositioning system of the calibration facility (step-by-step motor) and confirmed with a goniometer.

Figure 7 reveals again the wake-affected region in the angular interval between 10 and 40 deg. The general trend is opposite to the one analyzed in figure 6 , so the error associated to the flow angle retrievement turns out to be higher for yaw angles close to $0 \mathrm{deg}$, being progressively reduced as we move towards the angular limits of the calibration. Typical errors are found to be of $3 \mathrm{deg}$ at $\alpha=0 \mathrm{deg}$ and $1 \mathrm{deg}$ at $\alpha=-40 \mathrm{deg}$. Once again, higher error is observed for lower velocities than for higher velocities with respect to the calibration reference, although the difference is now really subtle. Under wake interferences, for the angle with the maximum wake interference $(\alpha=20$ deg), the error is progressively increased as the flow velocity slows down with the maximum error reaching $7 \mathrm{deg}$. Also note that for this particular angle, the errors are notably higher than any other angle considered.

All these considerations confirm, also for the flow angle retrievement, that X-type probes should not be operated when the incident flow angle induces wake interference effects on the wires. 
$120^{\circ}$ DHW X-probe

Angular range with and without interference effects

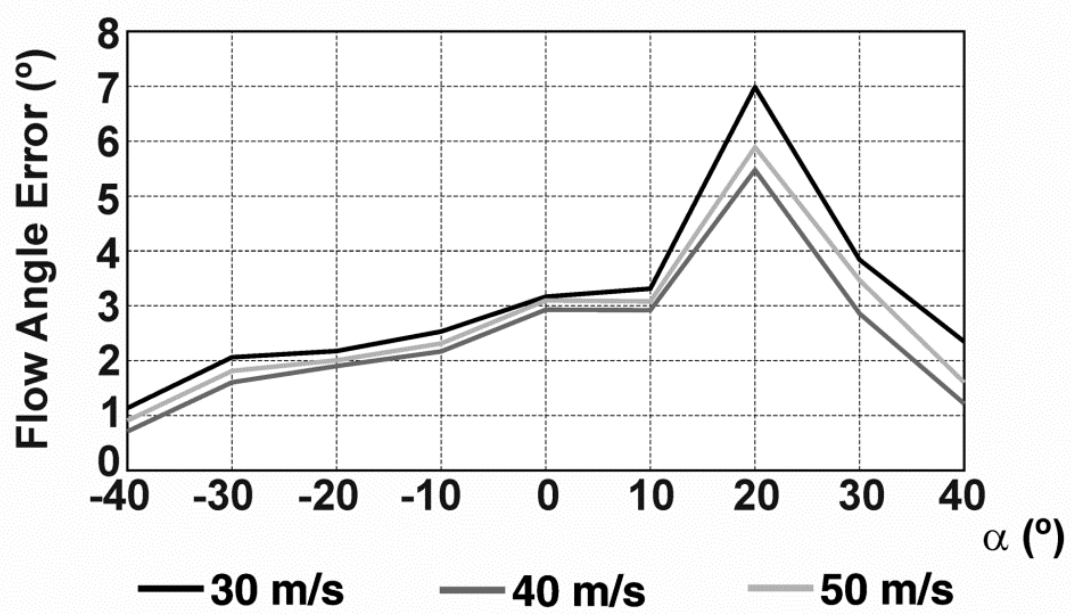

Figure 7. Error associated to the determination of the flow angle at different operating conditions.

\section{4.- Effect of wake interference on the estimation of turbulence intensity}

Additionally, the impact of measuring with the X-type probe in the wake-affected region of the calibration has been analyzed in terms of turbulence estimation. Notice that when wake interference effects are perturbing the inner wire, there must be superimposed an additional, fictitious turbulence provoked by the vortex shedding of the outer prongs, that may lead to an overestimation in the quantification of real turbulence levels.

In order to study such a hypothesis, additional tests at full high-frequency response of the Xtype probe were conducted. In particular, all the measurements have been sampled at $30 \mathrm{kHz}$ (close to the maximum frequency response of the probe), recording 10,000 instantaneous values during 0.3 seconds. Two different datasets have been considered for the analysis. Firstly, measuring in an incident flow with identical levels of inlet turbulence to those experienced during calibration (denoted as free-stream turbulence). Secondly, placing the probe in a flow with a slightly higher inlet turbulence (denoted as grid turbulence), which has been generated with a perturbing grid at the nozzle discharge of the facility. Measurements for impinging velocities of 30, 40 and $50 \mathrm{~m} / \mathrm{s}$ have been performed in both settings, ranging the yaw angle of the probe from -40 to $40 \mathrm{deg}$ in a $10 \mathrm{deg}$ interval. As before, the angular calibration at $40 \mathrm{~m} / \mathrm{s}$ has been systematically applied for data reduction of all the measured points.

Figure 8 shows power spectra of the time series corresponding for measurements obtained at 0 deg yaw angle (no interference with outer prongs) and 30 deg yaw angle (wake affected region) for an incident velocity of $40 \mathrm{~m} / \mathrm{s}$. Since they have been derived with the $40 \mathrm{~m} / \mathrm{s}$ calibration, they are free of inaccuracies related to Reynolds number deviations. Upper plot includes results from free-stream turbulence condition, while lower plot reproduces similar results for grid turbulence database. Both figures represent in the y-axis, in a logarithmic scale, the power spectrum density (PSD) of the velocity fluctuation from the instantaneous traces. These spectra have been postprocessed with a smoothed periodogram based on a parametric algorithm (autoregressive 800th order) to reduce spurious scatter. Additionally, a high-pass filter of $300 \mathrm{~Hz}$ has been used for signal conditioning to eliminate low-frequency oscillations of the supplying compressed air. 

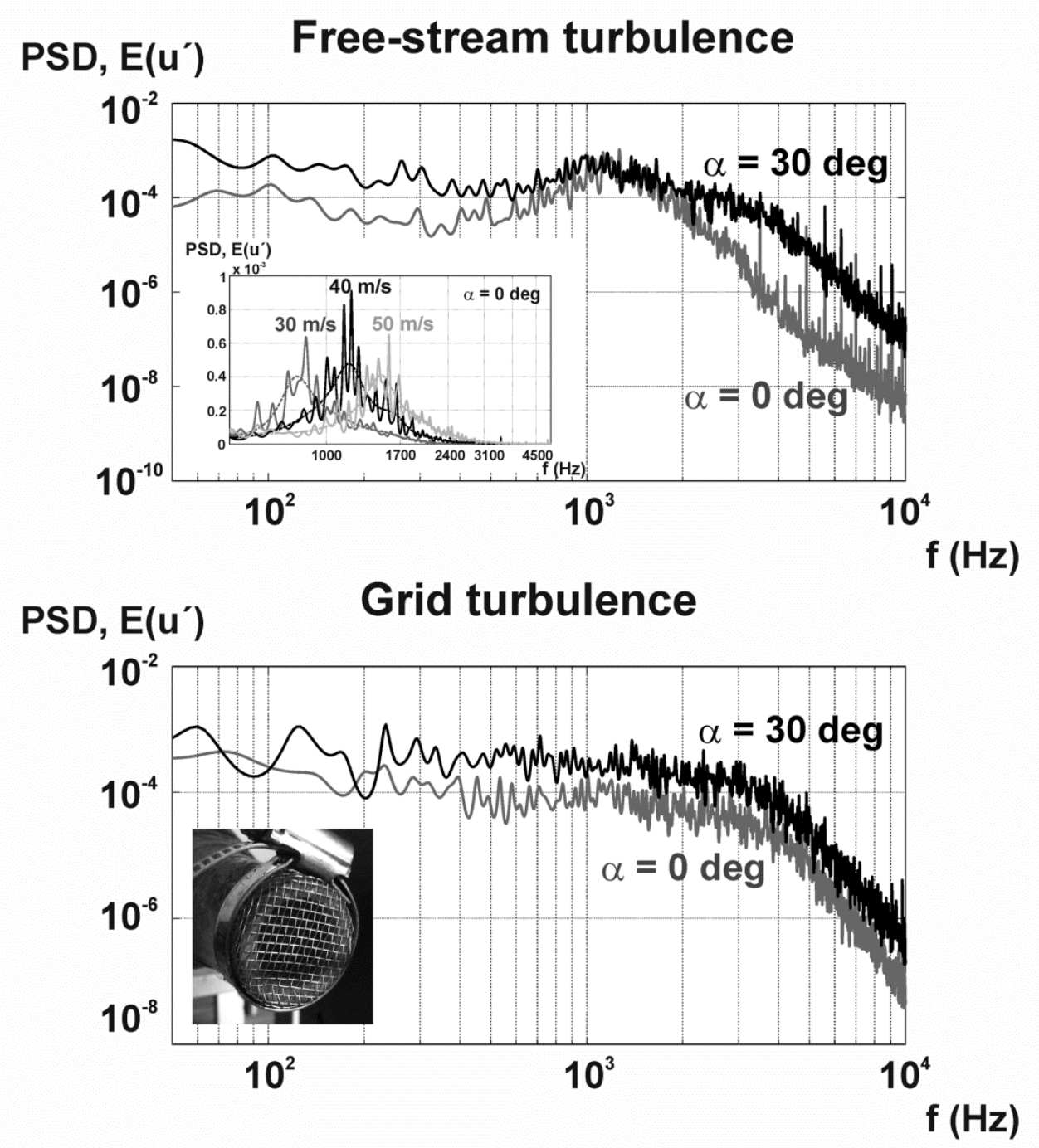

Figure 8. Power spectrum density (PSD) of the velocity fluctuation (incident flow of $40 \mathrm{~m} / \mathrm{s}$ ).

a) Free-stream turbulence conditions. b) Grid turbulence conditions.

Both situations (free-stream and grid turbulence) reveal that the energetic contents corresponding to wake-affected regions (30 deg) are higher for all the turbulent scales (throughout the whole frequency range) than out-of-wake locations ( $0 \mathrm{deg}$ ), clearly indicating that the probe is measuring an overestimated turbulence intensity at 30 deg yaw angle. It is also remarkable that for those spectra corresponding to free-stream conditions (figure 8 , top), there is a peak of turbulent kinetic energy around $1 \mathrm{kHz}$ for all the angular ranges tested. This phenomenon is reproduced for all the different velocities analyzed, as it can be seen in the small plot of the lower left corner in the figure. Note that this frequency signature is shifted along the $x-$ axis as the mean incident velocity is increased. The linear displacement indicates that this phenomenon must be related to vortex shedding in the nozzle discharge because when the grid is installed, this effect is vanished. In particular, considering a typical Strouhal number around 0.2, it has been estimated a $7 \mathrm{~mm}$ length scale as representative for vortex shedding, in reasonable accordance with the real blunt thickness of the nozzle endwall at the calibration facility.

Figure 9 (top) shows the angular distribution of turbulence levels (in percentage) for the incident flow angle of $40 \mathrm{~m} / \mathrm{s}$. This intensity has been computed from the instantaneous velocity fluctuation. Note that these results are again free of errors from Reynolds number deviations. Whatever configuration considered, wake-free regions (from -40 to $10 \mathrm{deg}$ ) present quite uniform, 
low values, ranging between 1.5 and 2\%. Conversely, from $10 \mathrm{deg}$ on, due to the effect of interference, there is a significant increase in the turbulence intensity, reaching up to $3.5 \%$ for extreme angles of $40 \mathrm{deg}$. It is also remarkable the slight difference between turbulence levels for both datasets at wake-free regions, as a direct consequence of the extremely thin grid employed in these measurements. These results confirm the overestimation in the measurement of the turbulence level when the probe is operated under wake-interference effects. Considering that hot-wire anemometry technique is widely used to characterize turbulent variables in real flows, it is quite reasonable to affirm that wake-affected regions are not valid ranges for measurements requiring high-frequency response.

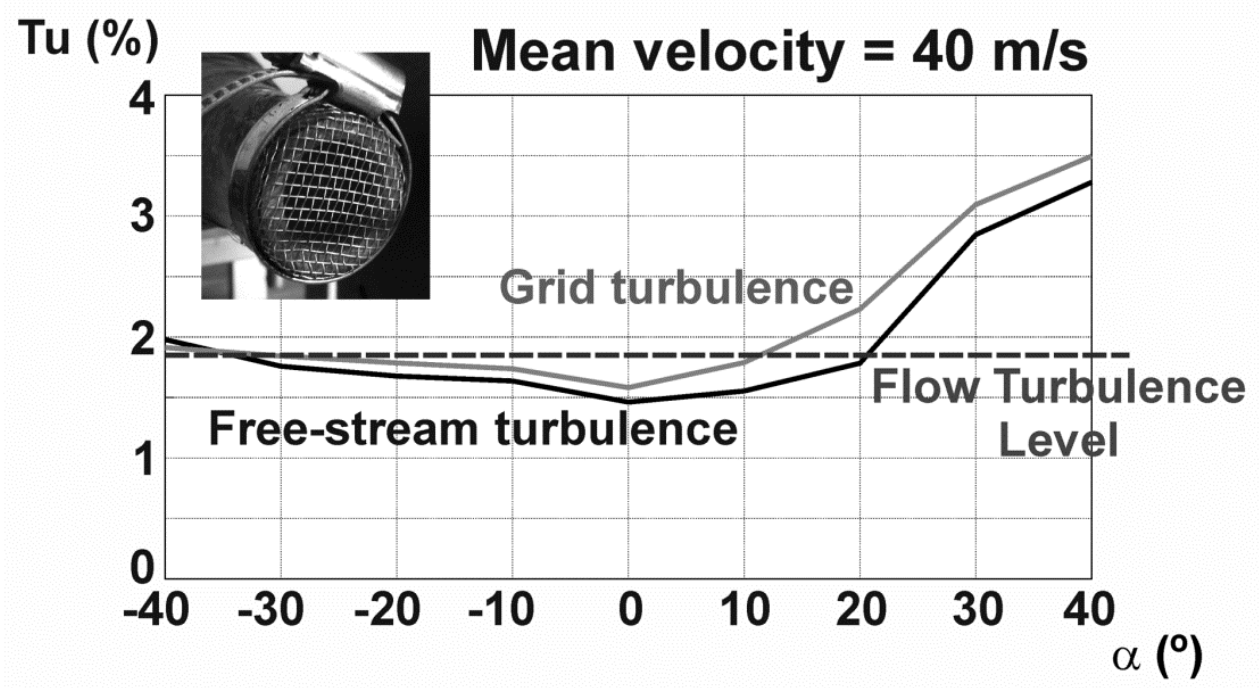

Tu (\%)

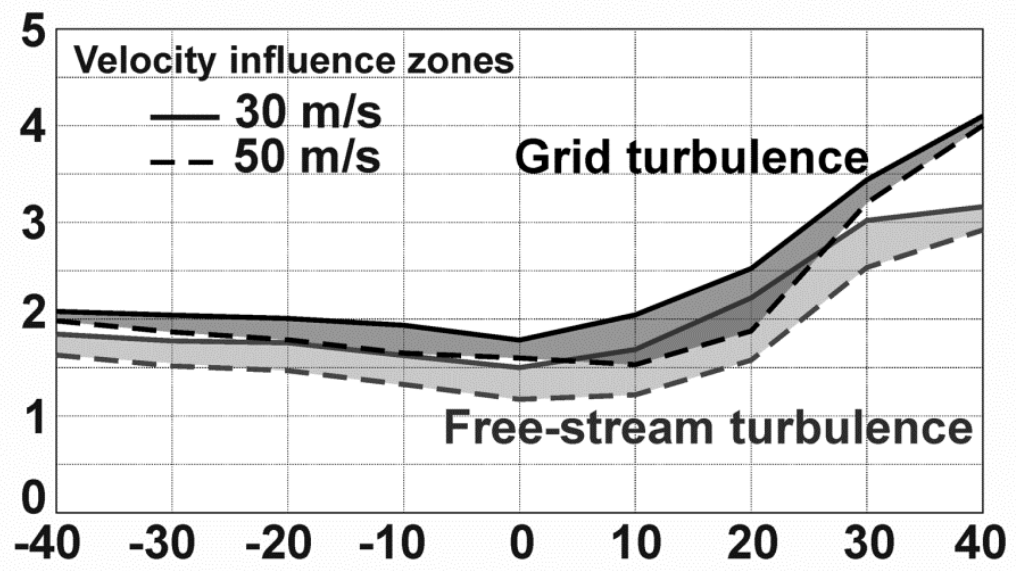

\section{$\alpha\left({ }^{\circ}\right)$}

Figure 9. Turbulence intensity as a function of the incidence angle.

a) Baseline conditions at $40 \mathrm{~m} / \mathrm{s}$ ). b) Deviations at different flow velocities.

Finally, figure 9 (bottom) shows the effect of Reynolds number deviations from calibration conditions over the turbulence intensities measured from the instantaneous velocity. The shaded regions represent the angular distribution of the dispersion of the results, when reducing data with out-of-velocity calibrations, which somehow corresponds to the maximum associated errors. Dark grey band identifies the dispersion in the turbulence level estimation for the grid turbulence setting, while light grey band represents dispersion associated to free-stream conditions. Obviously, higher intensity levels throughout the whole angular range are obtained for the 
perturbed conditions of the grid turbulence setting. Dashed lines are turbulence intensity levels for the $50 \mathrm{~m} / \mathrm{s}$ inlet flow, while solid lines represent those levels for the $30 \mathrm{~m} / \mathrm{s}$ case. The overall trend is that when the mean velocity is increased, the turbulence level seems to be progressively reduced. For no interference zones, the evolution of the turbulence intensity is similar for all the considered velocities, with maximum discrepancies of a $0.5 \%$. On the contrary, for wake-affected regions between 10 and $40 \mathrm{deg}$, changes in the intensity trends seem to be more pronounced with velocity variations, with typical errors in the order of $1 \%$. Hence, it is not recommended the use of data obtained under wake interferences to characterize turbulence levels.

In summary, the static calibration of X-type probes operating under wake-affection has revealed strong inaccuracies in the retrievement of the flow angle and velocity magnitude of steady turbulent flows. Additionally, the estimation of the turbulence intensities was also observed to be seriously compromised due to the introduction of fictitious turbulence provoked by the vortex shedding of the prongs under interference. To conclude with this section, Table 1 summarizes typical errors found in the present investigation for typical 120 deg X-type probes when operated under steady conditions.

Table 1. Errors in velocity, angle and turbulence intensity with typical 120 deg X-type probe for steady conditions.

\begin{tabular}{lcccc}
\hline \multirow{2}{*}{ Steady errors } & \multicolumn{2}{c}{ Wake-free region } & \multicolumn{2}{c}{ Wake interference (max) } \\
\cline { 2 - 5 } & $\begin{array}{c}\text { Calibration } \\
\text { velocity }\end{array}$ & $\begin{array}{c}\text { Out-of-velocity } \\
\text { calibration }\end{array}$ & $\begin{array}{c}\text { Calibration } \\
\text { velocity }\end{array}$ & $\begin{array}{c}\text { Out-of-velocity } \\
\text { calibration }\end{array}$ \\
\hline Velocity (\%), $\boldsymbol{e}_{\boldsymbol{v}}$ & $1-3 \%$ & $2.5-5 \%$ & $10 \%$ & $13 \%$ \\
Angle (deg), $\boldsymbol{e}_{\boldsymbol{\alpha}}$ & $0.75-2.75 \mathrm{deg}$ & $1.25-3.5 \mathrm{deg}$ & $5.5 \mathrm{deg}$ & $7 \mathrm{deg}$ \\
Turbulence (points), $\boldsymbol{e}_{\boldsymbol{t}}$ & $0.25 \mathrm{p}$. & $0.5 \mathrm{p}$. & $1-1.5 \mathrm{p}$. & $2-2.5 \mathrm{p}$. \\
\hline
\end{tabular}

\section{4.- UNSTEADY PERFORMANCE FOR A SINGLE-STAGE, LOW-SPEED AXIAL FAN}

Because hot-wire anemometers are specifically employed to measure unsteady flows and turbulence, the present study has been extended to characterize the inaccuracy of DHW probes measuring under interference effects in the case of turbomachinery flows. In particular, the unsteady flow patterns within a low-speed axial fan have been considered here to quantify the discrepancies arising in the determination of the time-resolved structures of the flow (velocity, angle and turbulence intensities) when measuring with the X-type probe. Note that measurements obtained with the V-type are taken as the reference for the comparison (i.e., it is supposed that flow variables are correctly measured with the V-probe). Since both Z-type and V-type probes have presented analogous calibration coefficients (figure 5), we have employed intensively the Vtype probe for the experimental characterization of the flow fields in the turbomachine due to its higher compactness and simplicity to be manufactured and repaired. However, for validation, a reduced number of additional measurements were carried out with the Z-type probe in order to confirm the results given by the $\mathrm{V}$-probe. The match between the results provided by both $\mathrm{V}$ - and Z-type probes was quite satisfactory and it is overviewed later in figures 12, 14 and 15.

In the following, some details of the measuring database concerning the low-speed fan are given. Also, the basic flow structures and the flow patterns downstream of the stator and rotor are 
presented (all the maps measured with the V-type probe). Finally, discrepancies obtained in the flow variables with the $X$-probe are quantified, especially in those locations with time-averaged flow angles in the range of the wake interference (15-30 deg).

\section{1.- Measuring database and time-averaged flow structures.}

An experimental database has been obtained using the V-type probe to describe the unsteady flow patterns in a single-stage low-speed axial fan with a rotor-stator (R-S) configuration. The axial blower consists of a 9-bladed rotor with a 13-vaned stator operated at $2400 \mathrm{rpm}$ (BPF = $360 \mathrm{~Hz}$ ) and tip and hub diameters of 820 and $380 \mathrm{~mm}$ respectively. The design conditions for the fan are $16.5 \mathrm{~m}^{3} / \mathrm{s}$ of delivered flow rate and $1.2 \mathrm{kPa}$ of pressure rise. The anemometric probe, with an estimated frequency response of $30 \mathrm{kHz}$, was intensively employed rotor downstream and within interrow regions in a number of traverse measuring windows of stator pitch periodicity. The probe was placed at half a chord from the trailing edge for each row. The radial discretization of the windows ranged from 15 to 23 spanwise locations, whereas the tangential discretization ranged from 15 to 28 pitchwise positions (see figure 10). For this particular study, only nominal conditions have been analyzed. More geometrical and operational details can be found in [22].

With respect to the acquisition parameters, a temporal resolution of $N=100$ samples per blade passing period was fixed, resulting in a sampling rate of $36 \mathrm{kHz}$ (a hundred times the BPF) and 2.5 points per degree at the rotor outlet. Also, for every velocity trace, up to $M=200$ blade passing periods (22 revolutions for the 9-bladed rotor @2400 rpm) were approximately recorded, so a total number of $2 \cdot 10^{4}$ samples were stored during $0.544 \mathrm{~s}$ at every measuring location.

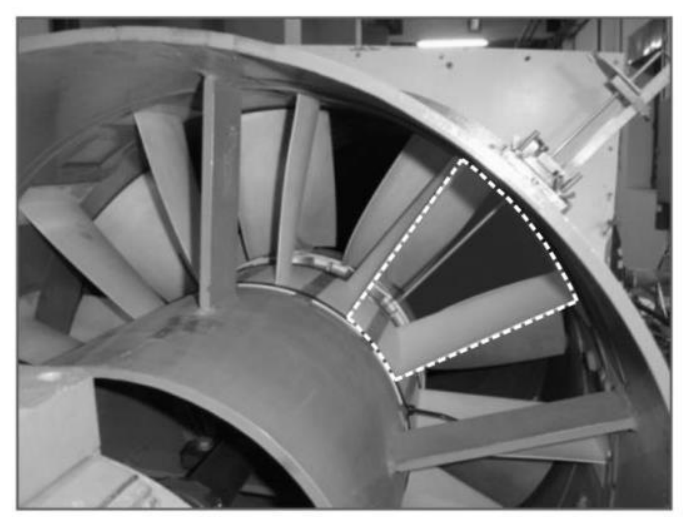

Experimental facility. Traverse windows (DHW probe at measuring location)

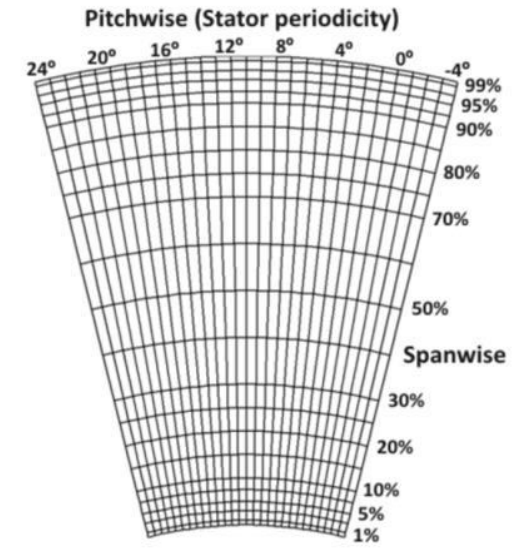

$[23 \times 29]$ spatial resolution

Figure 10. Measuring windows and spatial discretizations of the low-speed axial fan database.

Figure 11 shows averaged flow patterns throughout the stage of the axial fan, in terms of flow angle, measured with the $V$-type probe. The contour maps on the left present the time-averaged angle at nominal conditions for both relative (rotor) and absolute (stator) frames; while the right plot provides the pitch-averaged distributions along the spanwise direction (i.e., the circumferential averaged values). At the rotor outlet, the rotor wake is clearly visible from hub to tip in the angle distribution, with time-averaged values in the range of 20-25 deg. In addition, the tip vortex is also identified (and large overturning regions at $30 \%$ of the span) with large angle values (up to $45 \mathrm{deg}$ ), close (but still valid) to the upper limit (50 deg) of the probe operative range. Alternatively, at the stator outlet, just in the inner half of the measuring window, there are 
significant values of the flow angle (in the 20 deg range). Moreover, only the outer region (from $70 \%$ of the span on) presents low values (between 0 and $5 \mathrm{deg}$ ) because of the flow realignment into the axial direction induced by the stator. It is evident from the results that more than a $30 \%$ of the inner span of the stator vanes is not working properly because of the excessive residual overturning of the flow. With respect to the right plot, concerning the pitch-averaged (or circumferential-averaged) representation of the flow angles at both rotor and stator outlets, it is clearly manifested that the angle rotor downstream is within the range of the wake-interference regions presented by typical 120 deg X-type probes. This implies that all unsteady and turbulent measurements rotor downstream must be carefully analyzed when measuring with the X-probe. On the contrary, almost the whole spanwise distribution at the stator exit is out of the interference region, thus suggesting that $\mathrm{X}$-probe measurements should be free of errors there.
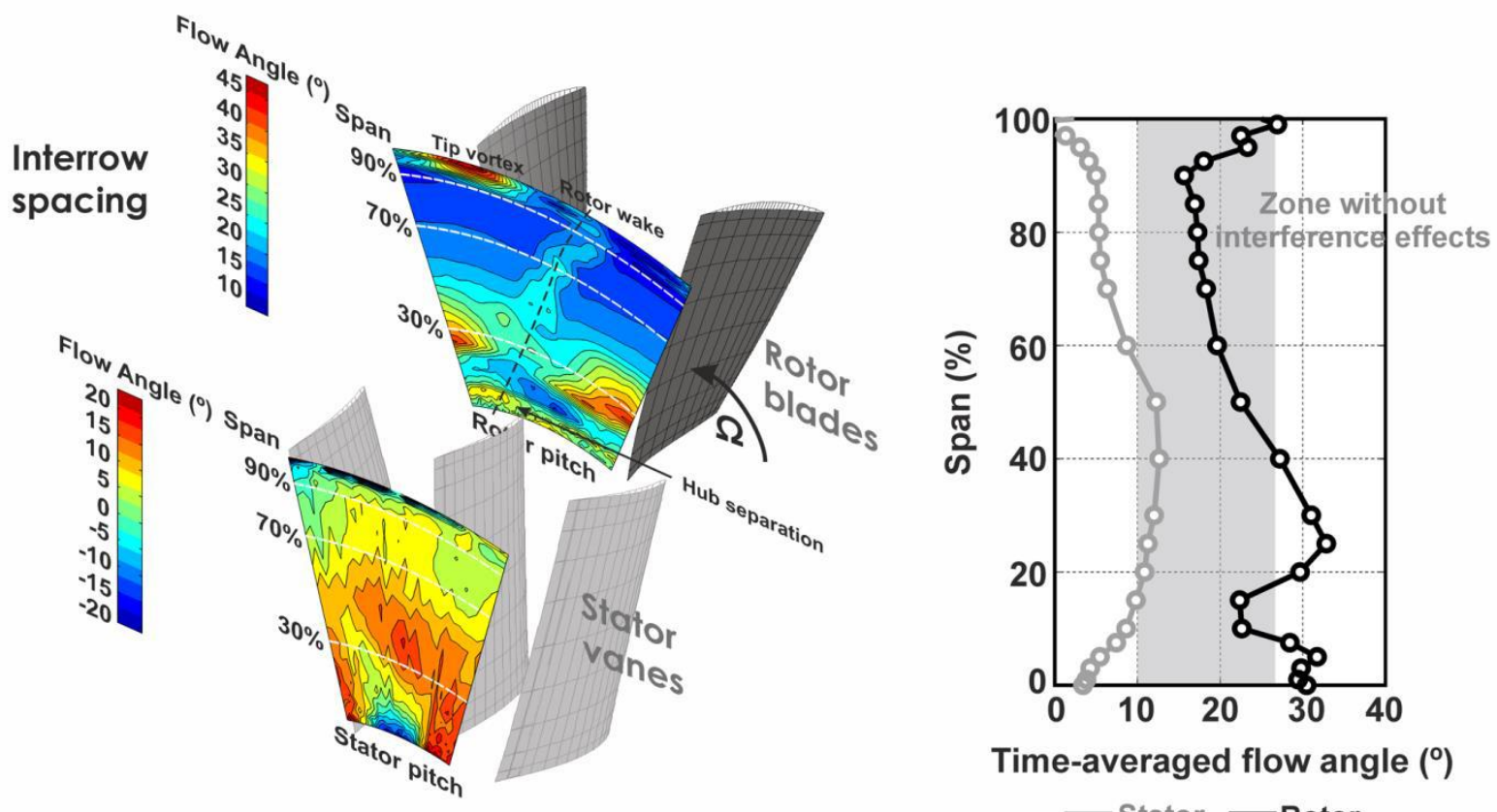

Time-averaged flow angle $\left(^{\circ}\right)$

- Stator -Rotor

Figure 11. a) Time-averaged flow angle at rotor (relative) and stator (absolute) outlets.

b) Pitch-averaged distributions rotor and stator downstream.

\section{2.- Error in the unsteady determination of flow angle and velocity downstream of the rotor.}

The inaccuracies in the unsteady measurement of the velocity and flow angle in the rotor outlet using the $X$ probe is described in figure 12. For that purpose, the time-resolved evolution of the axial velocity and flow angle at three different spanwise locations (30\%, $70 \%$ and $90 \%$ of the span) are represented for the three different probes analyzed. In the $x$-axis, time has been normalized by the blade passing period, $T_{R}$. Since all these measurements have been phase-averaged using 200 blade events, all the turbulent scales are filtered and only the deterministic fluctuations are preserved. Note that all these measurements present flow angles within the angular range susceptible for wake interference in the X-type probe. As expected, results from both V-type (gray line) and Z-type (dark line) probes are pretty close. On the contrary, all the traces for the X-type probe (dashed black line) differ substantially from those coming from the free-wake probes. In particular, velocity signals report significant deviations, always underestimating the real values. 
Table 2 reports such differences in average, in the range of 8-10\%, except close to the hub where low velocities raise the inaccuracies up to a $24 \%$. Concerning the flow angle, the X-probe overestimates the real values, providing overall errors in the range of 6.6-9.4 deg.

\section{$30 \%$ Span}
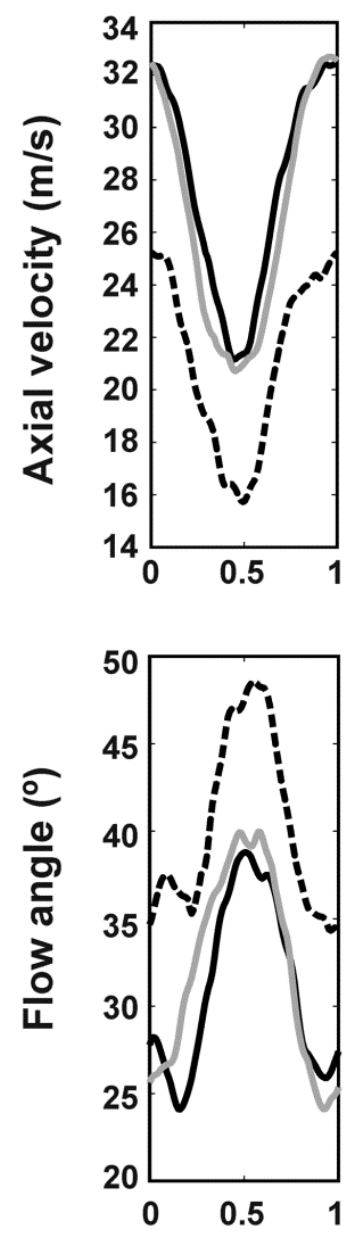

\section{- V-probe}

$70 \%$ Span
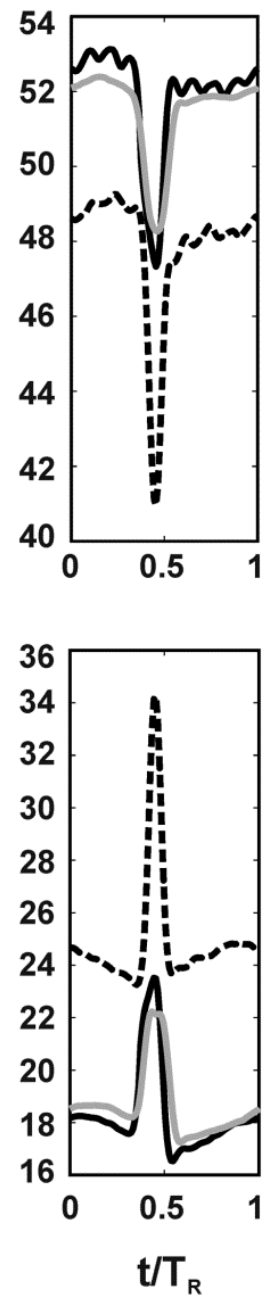

Figure 12. Unsteady, time-resolved evolutions of the velocity magnitude (a) and the flow angle

(b), obtained with wake-free ( $\mathrm{V}$ - and Z-types) and wake-affected (X-type) probes.

Table 2. Averaged errors in velocity, angle and turbulence intensity with typical 120 deg X-type probe for unsteady conditions rotor downstream.

\begin{tabular}{lccc}
\hline $\begin{array}{l}\text { Unsteady errors } \\
\text { (time-averaged) }\end{array}$ & $\mathbf{3 0 \%}$ Span & 70\% Span & 90\% Span \\
\hline Velocity (\%), $\overline{\boldsymbol{e}}_{v}$ & $23.9 \%$ & $7.8 \%$ & $10 \%$ \\
Angle (deg), $\overline{\mathbf{e}}_{\alpha}$ & $9.3 \mathrm{deg}$ & $6.6 \mathrm{deg}$ & $9.4 \mathrm{deg}$ \\
Turbulence (points), $\overline{\mathbf{e}}_{\boldsymbol{t}}$ & $6.7 \mathrm{p}$. & $1.75 \mathrm{p}$. & $1.64 \mathrm{p}$. \\
\hline
\end{tabular}


In addition to the time-averaged errors reported in table 2, the time-resolved evolution of the error in the velocity and angle determination at the rotor outlet is also of interest. The temporal description of the $\mathrm{X}$-probe errors helps to understand the real significance of the unsteady conditions on the prediction of the flow variables with probes operated under interference.

Figure 13 (left) reveals the spatial distribution of the error in the velocity retrieved with the Xprobe compared to the real values given by the V-probe. Larger discrepancies arise in those regions with large velocity deficits (i.e, the rotor wake and the tip vortex). Note that end-wall boundary layers in both hub and tip sections have been obviated in the analysis because of the inherent three-dimensionality of the flow. In the rotor wake, the instantaneous flow angle is easily 20-30 deg (figure 11.a), leading to a full interference in the X-probe. Consequently, the error is maximized, reaching up to a $20 \%$ as shown in the left plot. This error is significantly higher with respect to the steady levels reported in the steady calibration (10-13\%, table 1), revealing the real impact of unsteady (instantaneous) conditions on the errors.

Similar analysis can be done for the flow angle in the case of the right map in figure 13. Rotor wake is recovered once again in the error map for the flow angle, with a characteristic overestimation of the angle in the range of 16-18 deg with respect to the real values. This instantaneous errors associated to the rotor wakes are practically twice the characterized errors of the steady calibration (5.5-7 deg) shown in table 1.
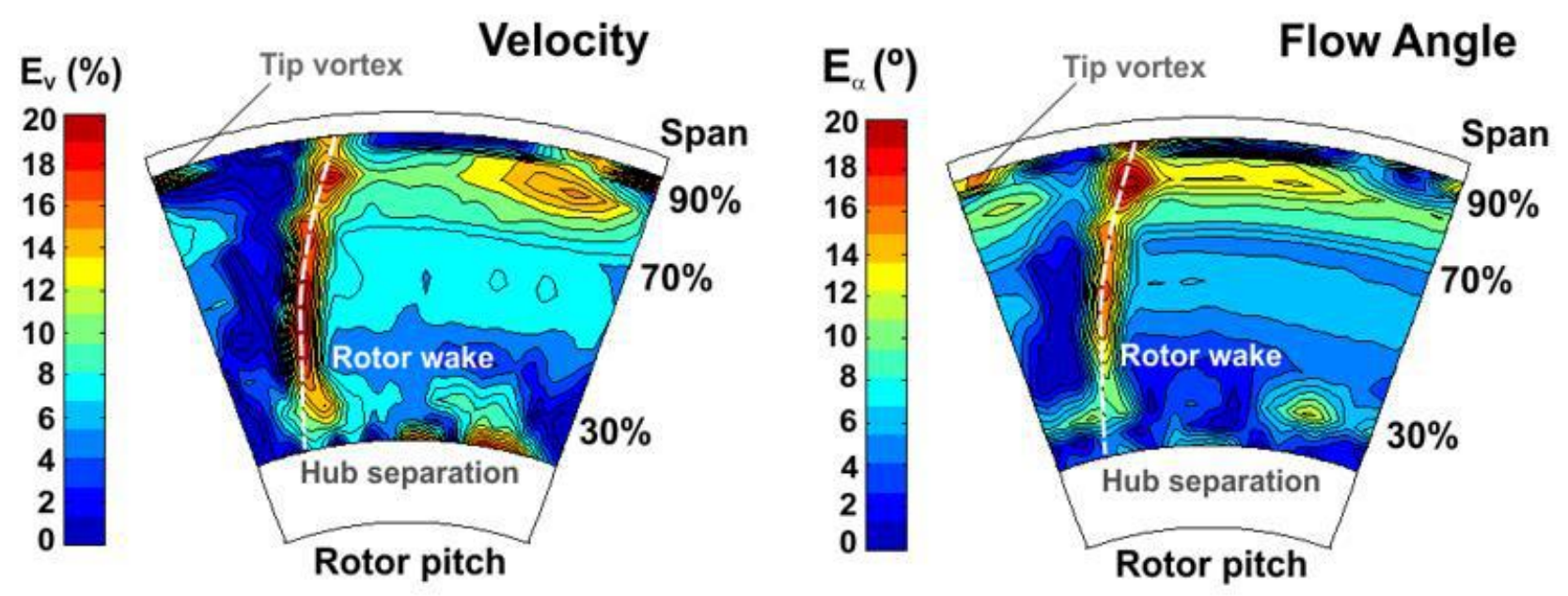

Figure 13. Maps of error in the determination of the velocity magnitude and flow angle with an Xtype probe operated under interferences in unsteady conditions at the rotor outlet.

\section{3.- Error in turbulence intensity downstream of the rotor}

The error in the determination of the turbulence intensity with the X-probe is shown in figure 14. In the left map, a representation of the real turbulence levels at the rotor outlet measured with the V-probe is firstly given. The turbulence intensity is defined as the RMS value of the turbulent fluctuations with respect to the time-averaged velocity in every measuring point as usual. The large regions of separation close to the hub involve significant levels of turbulence, up to a $30 \%$. Also the tip vortex is associated with high levels of turbulence. At the right, the results provided by each of the measuring probes are given now in terms of turbulence level for three 
different radial positions ( $30 \%, 70 \%$ and $90 \%$ of the span) in the stacked plots of the figure. V-type and Z-type probes present once again similar levels of turbulence, especially in the calmed regions out of the rotor wake. On the contrary, the X-probe overestimates the turbulence levels, as already devised in the case of the steady calibrations. At the wake, the double peak corresponds to the two viscous shear layers. Table 2 also lists averaged deviations of the X-probe results from those given by the $\mathrm{V}$-probe. Because turbulence intensities are computed using the local velocities as the reference, it is also expectable to observe large deviations in the turbulence intensity in the hub region (up to 6.4 points). Similarly, in the outer half of the window, averaged errors in the range of 1.5-1.75 points are quite similar to those reported in the steady calibrations. This means that in average, $\mathrm{X}$-probe errors are coincident for both steady and unsteady situations, but local or instantaneous values may be even higher.

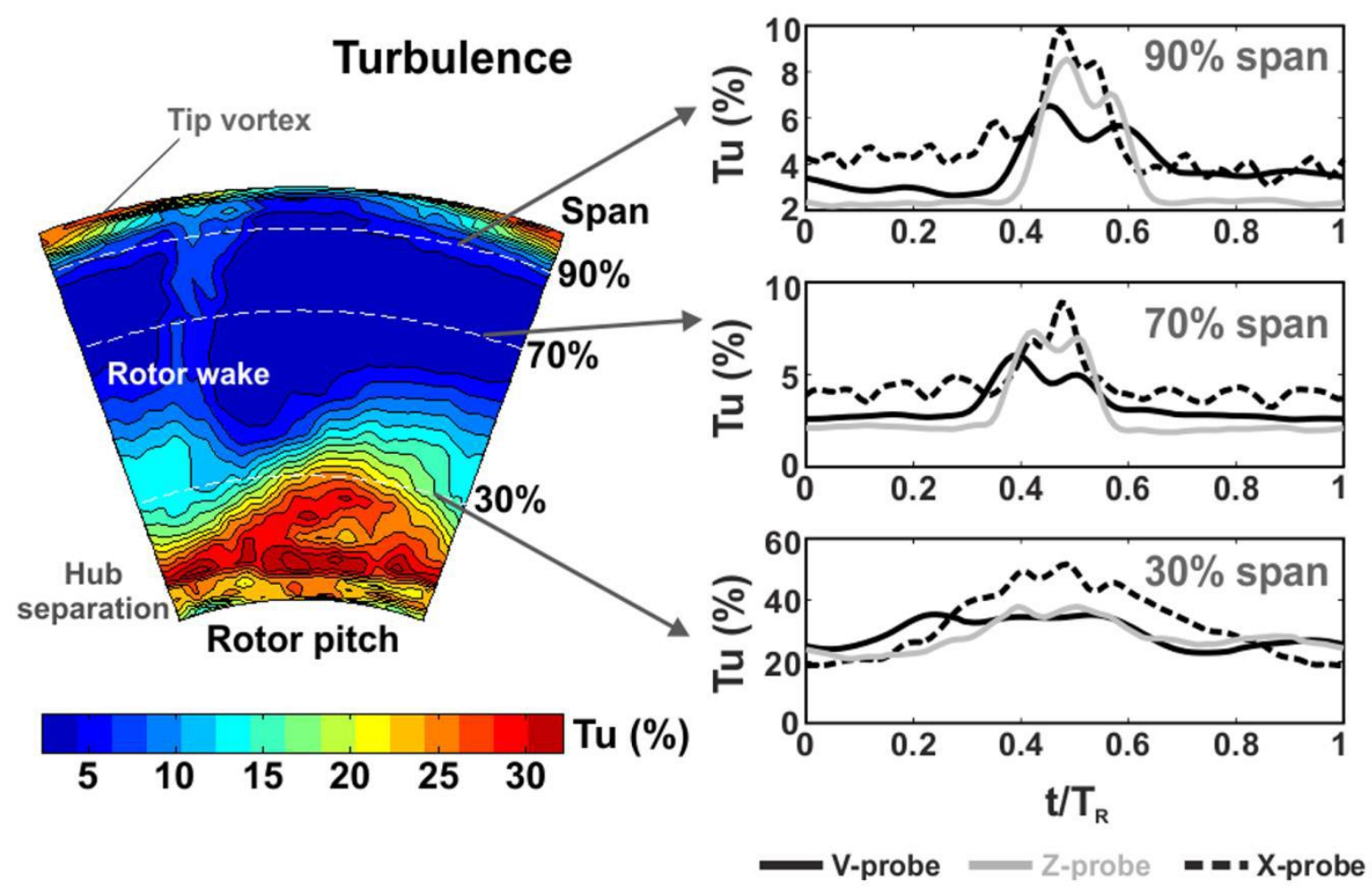

Figure 14. Maps of error in the determination of turbulence intensity with an X-type probe operated under interferences in unsteady conditions at the rotor outlet.

\section{4.- Discrepancies downstream of the stator}

To conclude this analysis, the unsteady behaviour of the X-probe is tested in a location that is not susceptible for wake interference, as in the case of the locations downstream of the stator. Figure 15 presents the temporal distributions of the velocity, flow angle and turbulence levels at $70 \%$ of the span at the rotor outlet. Here, the flow angle is clearly out of the wake interference region (the averaged flow angle is approximately $7 \mathrm{deg}$, as shown in figure 11.b). Moreover, the rotor wakes have been significantly mixed out, so they are much more diffused than in the interrow region (see for instance the velocity deficit in the top plot). With respect to the performance of the probes, it is clearly observed that the error in velocity, angle and turbulence is notably attenuated for the X-probe. The results reveal that the deviations are within 
characteristics uncertainties of this kind of DHW probes for unsteady flow measurements [19] (1-3 deg for flow angle and $1.5-3 \%$ in velocity).

\section{$70 \%$ stator span}
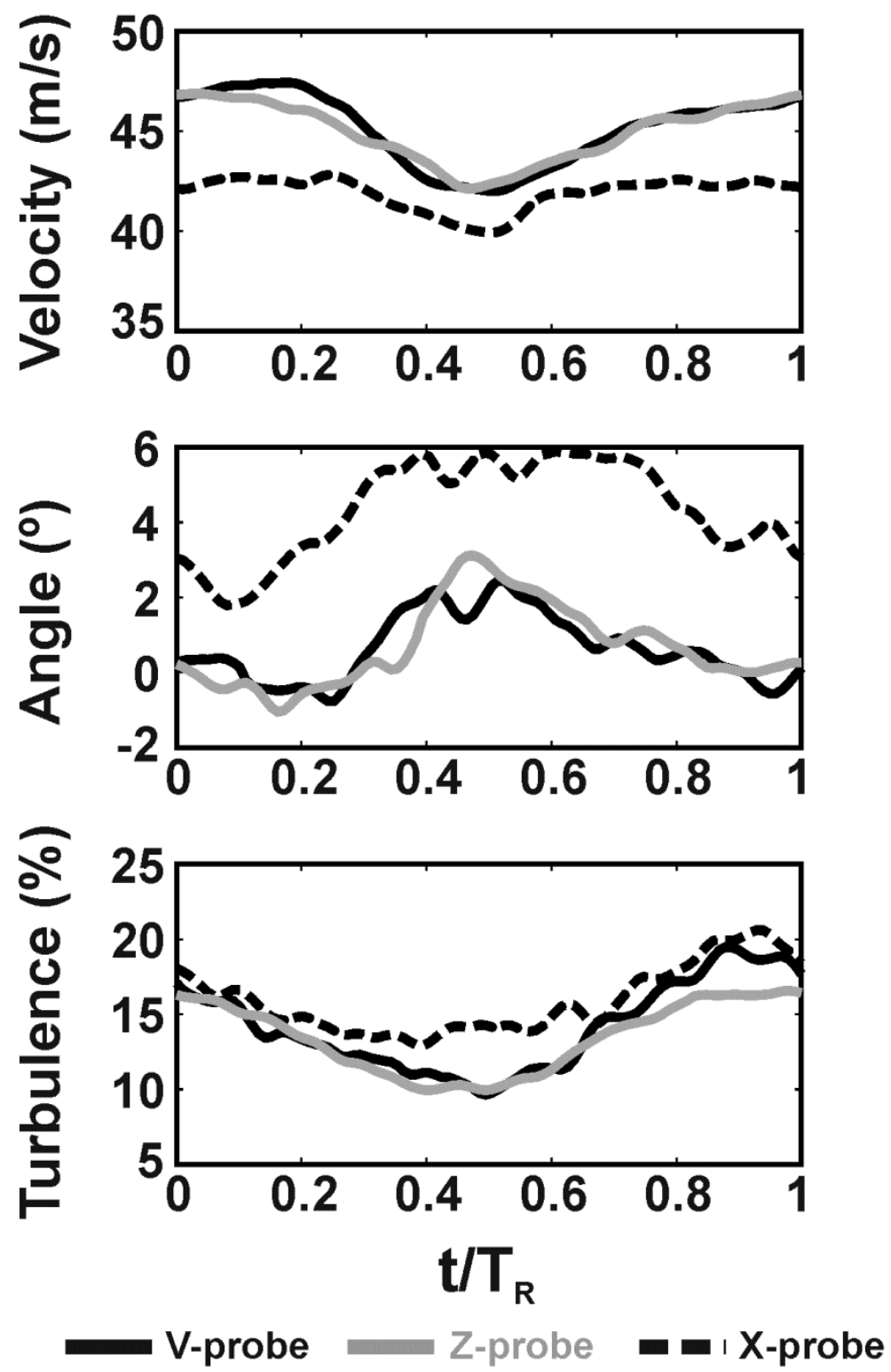

Figure 15. Unsteady, time-resolved evolutions of the velocity magnitude (a), flow angle (b), and turbulence intensity (c) obtained with wake-free (V-and Z-types) and wake-affected (X-type) probes at $70 \%$ of the span, stator downstream.

\section{5.- CONCLUSIONS}

In the present investigation, the measurement deviation caused by hot-wire probes operated under interference effects has been studied and evaluated, especially in the case of unsteady flows in turbomachinery environments. A classic X-array probe with wake interference effects in its operative range has been calibrated and employed intensively to compare its performance (static and dynamic) with respect to other probes without interference. In particular, Z-type and V- 
type designs have been developed as wake-free interferences probes to quantify this detrimental phenomenon in both steady and unsteady conditions.

It has been confirmed that wire-prong interferences produce a negative impact on the angular calibration of the probes, reducing significantly their operative angular range. Probes without interference effects may present operative angular ranges up to 35 deg higher than classic X-type probes. This increment can be especially desirable for the characterization of flows with large angular variations (i.e. time-averaged flow patterns in turbomachinery environments).

Furthermore, in the case of steady flows, the baseline deviation of the X-array probe in the measurement of the velocity magnitude, the flow angle and the turbulence intensity has been computed when measuring under wake interference conditions. Typical errors up to $10-13 \%$ in velocity, 5.5-7 deg in angle and 1.5-2.5 points overestimation in turbulence levels were observed. The performance of the probe to measure velocities out-of-calibration conditions was also explored showing a lower impact in the error levels.

Additionally, an in-depth study of the turbulence measurements has revealed that wake interference effects modify the whole turbulence spectrum, introducing an artificial fluctuation for all the turbulent scales. Once again, out-of-calibration conditions presents a reduced impact on the inaccuracies. Since hot-wire anemometers are conceived to provide a high-frequency response of the probe, wake interference effects are not acceptable for measuring turbulence.

Finally, the effect of measuring under prong-wire interference conditions in unsteady environments has been also explored. For that purpose, intensive measurements with the X-probe have been conducted within the rows of a single stage (rotor-stator), low-speed axial fan. Similar discrepancies (in average) to those observed in the steady tests have been reported when measuring the unsteady flow under interference conditions (rotor downstream). However, instantaneous deviations can be as much as twice higher, reaching up $20 \%$ in velocity error and 16-18 deg in flow angle when X-probes under interference are facing rotor wakes or vortical structures associated to the tip vortex. Turbulence intensities are also overestimated, in both calmed and perturbed regions, with the X-probe. Shear-free locations present discrepancies for the turbulence levels quite similar to those reported in the steady results, while turbulence intensity in the rotor wakes are excessively higher.

\section{ACKNOWLEDGEMENTS}

The authors acknowledge the support from the Research Project, "Communication by pressure waves in water pipes", ref. DPI-2012-36464, sponsored by the Spanish Ministry of Science and Innovation (MICINN).

\section{NOMENCLATURE}

$$
\begin{aligned}
\text { Acf } & =\text { Angular calibration coefficient }(-) \\
\mathrm{BPF} & =\text { Blade Passing Frequency }(\mathrm{Hz}) \\
\mathrm{CTA} & =\text { Constant Temperature Anemometer } \\
E & =\text { Output voltage }(\mathrm{V})
\end{aligned}
$$




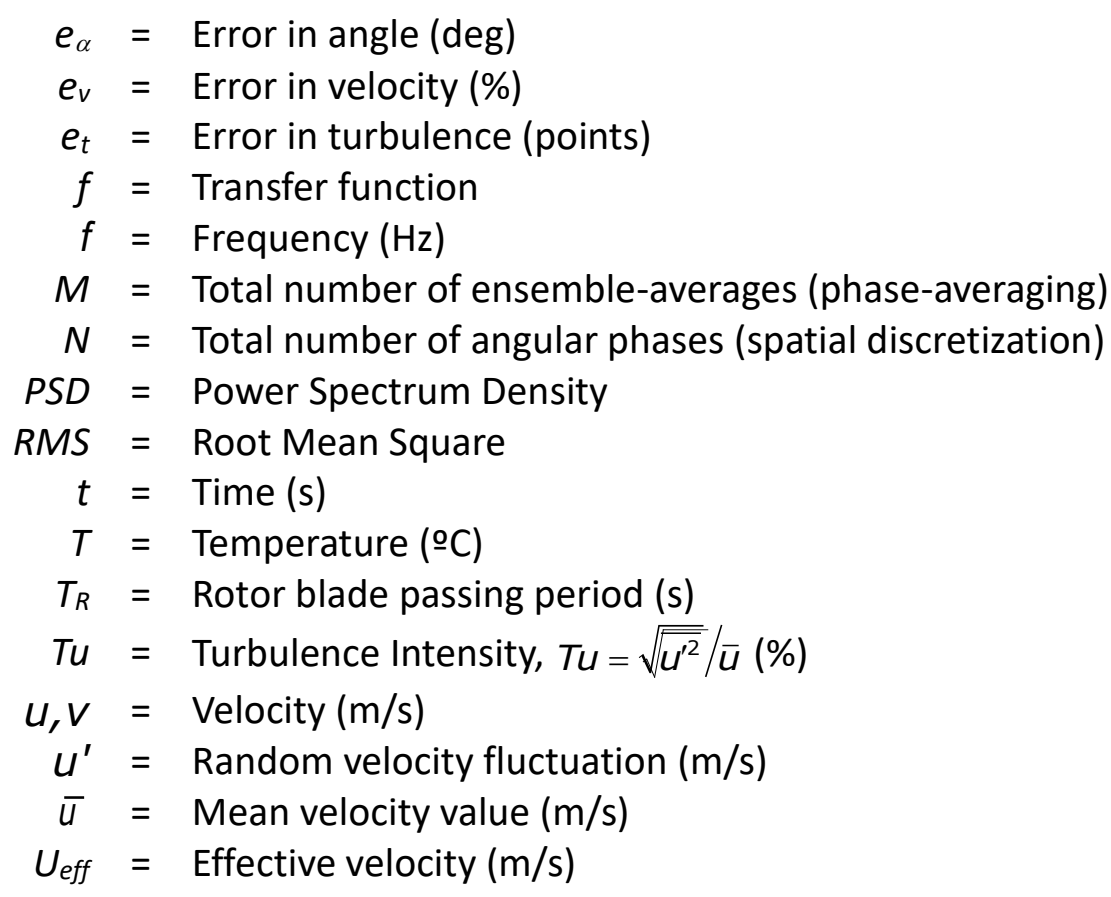

\section{Greek symbols}

$\alpha=$ Flow angle (deg)

\section{Superscripts and subscripts}

$$
\begin{aligned}
- & =\text { Time-averaging operator } \\
1,2 & =\text { Wire } 1,2 \\
a & =\text { Air } \\
\text { real } & =\text { Real value } \\
\text { meas } & =\text { Measured value } \\
w & =\text { Wire }
\end{aligned}
$$

\section{REFERENCES}

[1] Turan O and Azad RS. 1989. Effect of hot-wire probe defects on a new method of evaluating turbulence dissipation J. Phys. E: Sci. Instrum, 24, 254-61.

[2] Cameron JD, Morris SC, Bailey S and smith A. 2010. Effects of hot-wire length on the measurement of turbulent spectra in anisotropic flows. Meas. Sci. Technol, 21, 105407 (10 $\mathrm{pp})$.

[3] Sanitjai S and Goldstein RJ. 2004. Forced convection heat transfer from a circular cylinder in crossflow to air and liquids. International Journal of Heat and Mass Transfer, 47, 4795-4805.

[4] Jorgensen FE. 1996. The computer-controlled constant-temperature anemometer. Aspects of set-up, probe calibration, data acquisition and data conversion. Meas. Sci. Technol, 7, 13781387.

[5] Freymuth P. 1977. Frequency response and electronic testing for constant-temperature hotwire anemometers. Journal of Physics E, 10, 705-710.

[6] Freymuth P. 1997. Interpretations in the control theory of thermal anemometers. Meas. Sci. Technol, 8, 174-177. 
[7] Tropea C, Yarin A and Foss JF (Eds). 2007. Handbook of Experimental Fluid Mechanics. Springer-Verlag Berlin Heidelberg.

[8] Brunn HH, Nabhani N, Al-Kayiem HH, Fardad AA, Khan MA and Hogarth E. 1990. Calibration and analysis of $X$ hot-wire probe signals. Meas. Sci. Tecnol, 1, 782-785.

[9] Brunn HH, Nabhani N, Fardad AA and Al-Kayiem HH. 1990. Velocity component measurements by $X$ hot-wire anemometry. . Meas. Sci. Tecnol, 1, 1314-1321.

[10] Comte-Bellot G, Strohl A and Alcaraz E. 1971. On aerodynamic disturbances caused by single hot-wire probes. J. Appl. Mech, 38, 767-774.

[11] Strohl A and Comte-Bellot G. 1973. Aerodynamic effects due to configuration of X-wire anemometers. J. Appl. Mech, 40, 661-666.

[12] Adrian RJ, Johnson RE, Jones BG, Merati P and Tung ATC. 1984. Aerodynamic disturbances of hot-wire probes and directional sensitivity. J. Phys. E: Sci. Instrum, 17, 62-71.

[13] Wygnanski I and Ho C-M. 1978. Note on the prong configuration of an X-array hot-wire probe. Rev. Sci. Instrum, 49, 865-866.

[14] Bremhorst K and Gilmore DB. 1976. Comparison of dynamic and static hot wire anemometer calibrations for velocity perturbation measurements. J. Phys. E: Sci. Instrum, 9, 1097-1100.

[15] King LV. 1914. On the convection of heat from small cylinders in a stream of fluid: determination of the convection constants of small platinum wires with applications to hotwire anemometry. Philosophical Transactions of the Royal Society of London, 214, 373-432.

[16] Brunn HH, Khan MA, Al-Kayiem HH and Fardad AA. 1988. Velocity calibration relationships for hot-wire anemometry. J. Phys. E: Sci. Instrum, 21, 225-232.

[17] Brunn HH. 1971. Interpretation of a hot-wire signal using a universal calibration law. J. Phys. E: Sci. Instrum, 4, 225-232.

[18] Brunn HH and Tropea C. 1985. The calibration of inclined hot-wire probes. J. Phys. E: Sci. Instrum, 18, 405-413.

[19] Blanco-Marigorta E, Ballesteros-Tajadura R and Santolaria C. 1998. Angular range and uncertainty analysis of non-orthogonal crossed hot wire probes. Journal of Fluids Engineering, 120, 90-94.

[20] Stainback PC and Nagabushana KA. 1997. Review of hot-wire anemometry techniques and the range of their applicability for various flows. Electronic Journal of Fluids Engineering, 119.

[21] Lekakis I. 1996. Calibration and signal interpretation for single and multiple hot-wire/hot-film probes. Meas. Sci. Technol, 7, 1313-1333.

[22] Fernández Oro JM, Argüelles Díaz KM, Santolaria Morros C, Blanco Marigorta E. 2007. Unsteady flow and wake transport in a low-speed axial fan with inlet guide vanes. Journal of Fluids Engineering, 129, 1015-1029.

\section{LIST OF FIGURES}

Fig. 1. Typical X-type DHW probe with prong-wire interference.

Fig. 2. Schematic view of the probes: X-type, Z-type and V-type.

Fig. 3. a) Acquisition system: IFA 100 and NIDAQ devices. b) Nozzle and calibration facility.

Fig. 4. Angular distributions of wires output voltage and calibration coefficient for X-type probe.

Fig. 5. Angular distributions of wires output voltage (left) and calibration coefficients for Z-type and $V$-type probes (without wake interference, right).

Fig. 6. Error associated to the determination of the velocity magnitude at different operating conditions.

Fig. 7. Error associated to the determination of the flow angle at different operating conditions. 
Fig. 8. Power spectrum density (PSD) of the velocity fluctuation (incident flow of $40 \mathrm{~m} / \mathrm{s}$ ). a) Freestream turbulence conditions. b) Grid turbulence conditions.

Fig. 9. Turbulence intensity as a function of the incidence angle. a) Baseline conditions at $40 \mathrm{~m} / \mathrm{s}$ ). b) Deviations at different flow velocities.

Fig. 10. Measuring windows and spatial discretizations of the low-speed axial fan database.

Fig. 11. a) Time-averaged flow angle at rotor (relative) and stator (absolute) outlets. b) Pitchaveraged distributions rotor and stator downstream.

Fig. 12. Unsteady, time-resolved evolutions of the velocity magnitude (a) and the flow angle (b), obtained with wake-free (V-and Z-types) and wake-affected (X-type) probes.

Fig. 13. Maps of error in the determination of the velocity magnitude and flow angle with an $X$ type probe operated under interferences in unsteady conditions at the rotor outlet.

Fig. 14. Maps of error in the determination of turbulence intensity with an X-type probe operated under interferences in unsteady conditions at the rotor outlet.

Fig. 15. Unsteady, time-resolved evolutions of the velocity magnitude (a), flow angle (b), and turbulence intensity (c) obtained with wake-free (V- and Z-types) and wake-affected (Xtype) probes at $70 \%$ of the span, stator downstream. 
This document is a pre-print version of the scientific paper published by Elsevier. It has been released by the authors to fulfill all the publisher requirements established for Article Sharing: https://www.elsevier.com/about/policies/sharing

\section{(9) $\Theta \Theta \Theta$}

(C) 2019. This manuscript version is made available under the Creative Commons Attribution-NonCommercial-NoDerivatives 4.0 International License (CC-BY-NC-ND 4.0 license) http://creativecommons.org/licenses/by-nc-nd/4.0/ 